\title{
Sensitive, Subtype Independent HIV-1 PCR Assays for Assessment of Residual Viremia and Total HIV-1 DNA
}

\author{
Tomas Mellberg', Jon Krabbe ${ }^{2}$, Maria J Buzon ${ }^{3}$, Ulrika Noborg', Magnus Lindh' ${ }^{2}$, Staffan Nilsson ${ }^{4}$, Bo \\ Svennerholm ${ }^{2}$ and Magnus Gisslén ${ }^{1 *}$
}

${ }^{1}$ Department of Infectious Diseases, Sahlgrenska Academy, University of Gothenburg, Sweden

${ }^{2}$ Department of Clinical Virology, Sahlgrenska Academy, University of Gothenburg, Sweden

${ }^{3}$ Irsicaixa AIDS Institute, Badalona, Spain

${ }^{4}$ Mathematical Sciences, Chalmers University of Technology, Gothenburg, Sweden

*Corresponding author: Magnus Gisslén, Institute of Biomedicine, Department of Infectious Diseases, Sahlgrenska Academy, University of Gothenburg, SE 41685 Gothenburg, Sweden, E-mail: magnus.gisslen@gu.se

\begin{abstract}
Background: Correct measurements of residual viremia and reservoir size are crucial in HIV-1 eradication trials and there is a need for sensitive and automated assays. The increasing worldwide diversity of HIV-1 subtypes stresses the importance of subtype independent assays.

Methods: We added an ultra-centrifugation step to the COBAS AmpliPrep/COBAS TaqMan HIV-1 test, version 2.0 and evaluated assay sensitivity. A previously published method for quantification of total HIV-1 DNA was also evaluated. Nineteen clinical samples from virologically suppressed cART treated patients infected with various HIV-1 subtypes were analysed. The relationship between plasma HIV-1 RNA and HIV-1 DNA in Peripheral Blood Mononuclear Cells (PBMC) was studied.

Results: The HIV-1 RNA PCR assay reached a limit of quantification of 3 copies/ml of plasma. The total HIV-1 DNA assay allowed quantification to 3 copies/million cells with an intra- and inter-assay coefficient of variation of $13.2 \%$ and $23.2 \%$ respectively. All subtypes studied were quantified by the HIV-1 RNA PCR assay and the HIV-1 DNA assay quantified all subtypes except CRF_AE. No significant correlation was observed between plasma HIV-1 RNA and total HIV-1 DNA in PBMC.

Conclusions: By modification of a commercial assay, we achieved a sensitive quantification of plasma HIV-1 RNA that could be used to assess residual viremia. Sensitive quantification of total HIV-1 DNA in PBMC was also demonstrated. The assays were subtype independent to a large extent, making them useful for research and clinical use in settings where a variety of HIV-1 subtypes is present.
\end{abstract}

\section{Background}

Treatment of HIV-1 infection with effective combination Antiretroviral Treatment (CART) reduces plasma viral load to undetectable levels measured with clinical assays $[1,2]$. However, very stable viral reservoirs established early in infection are obstacles for eradication [35]. Precise measurements of the residual viremia that is released from these reservoirs combined with methods to estimate viral reservoir size are important in research for a cure of HIV-1. In recent years it has also been proposed that at least in some patients it may be clinically relevant to monitor viremia even below 50 HIV-1 RNA copies/ml $[6,7]$. The commercial assays of today have a limited sensitivity and a high variability in samples $<20$ to 40 copies $/ \mathrm{ml}$ depending on the assay [8].

The Single Copy Assay (SCA), a quantitative assay that can detect less than 1 copy of HIV-1 RNA/ml, has been widely used for research purposes in recent years [9-13]. This assay is labour-intensive and is currently limited to quantification of subtype B strains [14]. The methods need to be sensitive but also subtype-independent since many research and clinical environments are dealing with a diversity of HIV-1 subtypes. Several recent studies have reported increasing HIV-1 subtype diversity in different parts of the world, including Sweden and USA [15-18]. Previous reports have described in-house assays or modified commercial assays that in-

Citation: Mellberg T, Krabbe J, Buzon MJ, Noborg U, Lindh M, et al. (2017) Sensitive, Subtype Independent HIV-1 PCR Assays for Assessment of Residual Viremia and Total HIV-1 DNA. Int J Virol AIDS 4:030. doi.org/10.23937/2469-567X/1510030

Received: April 07, 2017: Accepted: June 29, 2017: Published: July 01, 2017

Copyright: (C) 2017 Mellberg T, et al. This is an open-access article distributed under the terms of the Creative Commons Attribution License, which permits unrestricted use, distribution, and reproduction in any medium, provided the original author and source are credited. 
crease their sensitivity [19-21]. For example, Havlir, et al. increased the sensitivity by using ultra-centrifugation prior to amplification with the Roche Amplicor Ultrasensitive assay [19]. Another, more recent study showed a single-copy sensitivity by increasing the sample volume, and adding an ultra-centrifugation step to the Abbott Real Time assay [21]. However, information about subtypes were lacking in these studies. Modified commercial assays have the advantage of wide availability, being extensively tested, standardised and automated. It should also be possible to detect different subtypes with good reproducibility using these assays. The COBAS Taqman HIV-1 test, version 2.0 from Roche (AS-CT2) has a reported lower limit of detection than the Amplicor and Abbott Real Time Assay [22,23], and the advantage of targeting both gag and LTR, which could increase the accuracy of the viral load determination and the spectrum of quantifiable HIV-1 isolates.

A possible strategy for HIV-1 eradication is to reactivate latent virus and thereby accelerate viral clearance in the presence of cART [24-28]. In studies evaluating effects on the latent reservoir it is necessary to use methods that are able to accurately measure the fraction of replication-competent virus in the reservoir. Currently, the Infectious Units per Million Cells (IUPM) assay is considered the most sensitive and precise assay for measuring HIV-1 viral reservoirs [29]. This assay is however labour intensive and it also has a high variance, a feature making it difficult to measure small differences in replication-competent virus with accuracy. Furthermore it seems to underestimate the actual replication-competent viral reservoir [30]. The measurement of total HIV-1 DNA (linear and circular non-integrated and integrated) and proviral HIV-1 DNA (integrated) has been proposed as surrogate measures of the viral reservoir [31-33]. Since linear unintegrated HIV-1 DNA has a short half-life in vitro and circular HIV-1 DNA is considered being produced during active replication, it is reasonable to assume that most of the HIV-1 DNA will be in an integrated form if replication is halted by cART for a long period of time. Measures of total and proviral HIV-1 DNA have therefore been proposed to be interchangeable surrogates for reservoir size [31]. However, these methods still have the disadvantage of measuring also non-viable virus and thereby risking overestimating the actual viable viral reservoir.

In this study, we added an ultra-centrifugation step prior to the routine RT-PCR with AS-CT2, to enable subtype independent quantification of residual viremia. We also evaluated an assay for ultrasensitive measurement of total HIV-1 DNA in Peripheral Blood Mononuclear Cells (PBMC). Clinical performance of both assays was evaluated in a correlation study between total HIV-1 DNA and plasma HIV-1 RNA in patients on long-term effective cART with different HIV-1 subtypes.

\section{Materials and Methods}

\section{Clinical specimens}

All clinical samples were collected from the Department of Infectious Diseases at Sahlgrenska University Hospital in Gothenburg, Sweden. Nineteen patients who had been on CART for more than 1 year and who had suppressed viral load in plasma (< 50 HIV-1 RNA copies/ml for a median of 54 months), were included in the study. All patients but two had undetectable HIV-1 RNA at study entry (measured by AS-CT2). Eleven patients were infected with HIV-1 subtype B. Four patients

Table 1: Characteristics of 19 clinical samples.

\begin{tabular}{|c|c|c|c|c|c|c|}
\hline Clade & $\begin{array}{l}\text { Pre-treatment HIV-1 } \\
\text { RNA (copies } / \mathrm{mI})^{*}\end{array}$ & 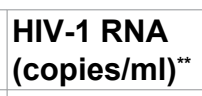 & $\begin{array}{l}\text { HIV-1 DNA total } \\
\text { (copies/million cells) }\end{array}$ & $\begin{array}{l}\text { HIV-1 RNA < } 50 \\
\text { copies (months) }\end{array}$ & $\begin{array}{l}\text { CD4 }^{+} \text {T-cell } \\
\text { nadir }\end{array}$ & $\begin{array}{l}\text { CD4 }{ }^{+} \text {T-cell } \\
\text { count }\end{array}$ \\
\hline B & 28755 & Det & 6.5 & 99 & 40 & 200 \\
\hline B & 926000 & Neg & Neg & 91 & 20 & 340 \\
\hline B & 76901 & 11.5 & 26.7 & 102 & 150 & 660 \\
\hline B & 293000 & 5.2 & 3.1 & 39 & 300 & 780 \\
\hline B & 20600 & 2.8 & 79.1 & 57 & 150 & 650 \\
\hline B & 1000001 & Det & 0.8 & 39 & 1 & 360 \\
\hline B & 784000 & 7.7 & 26.8 & 92 & 90 & 840 \\
\hline B & 9447 & Neg & 16.2 & 52 & 160 & 860 \\
\hline B & 74135 & 34.5 & 59.7 & 33 & 100 & 170 \\
\hline B & 41264 & Det & 1.4 & 120 & 200 & 780 \\
\hline B & 1800000 & Det & 15.4 & 8 & 210 & 400 \\
\hline C & 84200 & Neg & 24 & 33 & 170 & 360 \\
\hline C & 42000 & 2.3 & 11 & 71 & 54 & 310 \\
\hline C & 40073 & $\mathrm{Neg}$ & 3.3 & 98 & 130 & 540 \\
\hline C & 37900 & $\mathrm{Neg}$ & 17.8 & 16 & 170 & 500 \\
\hline $\mathrm{D}$ & 62100 & 9.7 & 1.5 & 5 & 10 & 610 \\
\hline $\mathrm{F}$ & 750000 & 3.4 & 155.6 & 28 & 130 & 190 \\
\hline CRF01_AE & 750000 & Det & Neg & 58 & 160 & 630 \\
\hline CRF01_AE & 448000 & 5.9 & $\mathrm{Neg}$ & 98 & 30 & 370 \\
\hline
\end{tabular}

Det: Detected but not quantified; Neg: Negative; *The COBAS Taqman HIV-1 test, version 2.0 (Roche); **Ultrasensitive HIV-1 RNA assay. 
were infected with subtype $C$, two patients with subtype CRF-AE, one patient with subtype $D$, and one was determined as probably subtype $F$, according to the HIVPOL subtyping algorithm of Stanford (HIV drug resistant database HIVDB.standford.edu) (Table 1). For statistical calculations in the correlation study, detected but not quantifiable samples were denoted as the mean value in the range between 0 copy/ml and the suggested limit of quantification derived from the sensitivity panels for each assay. Negative samples were denoted as 0.1 copies/ml for HIV-1 RNA and 0.1 copies/million cells for HIV-1 DNA.

For the low-copy-number HIV-1 RNA panel we used two different plasma samples from individuals with a known high viral load in plasma. For assessment of reproducibility and limit of detection of the real time PCR for quantification of total HIV-1 DNA we used two different samples from individuals with subtype $B$ viral clades with known high viral loads in plasma.

Low-copy-number HIV-1 RNA measurement panels: A plasma sample with viral load of 268,325 HIV-1 RNA copies/ml was diluted in HIV-seronegative plasma to give the estimated HIV-1 RNA concentrations of 2683, 537, 107, 26.8, 5.4 and 2.7 copies/ml (Panel 1). A second plasma sample was treated in the same way to give the estimated HIV-1 RNA concentrations of 27, 9, 3, 1 copies/ml (Panel 2). The initial viral load was determined by standard PCR with AS-CT2 according to the manufacturer. The total SD in log in this range is reported as 0.06 and total log normal CV $15 \%$ according to the manufacturer. The dilutions were stored in $50 \mathrm{ml}$ tubes and frozen at $-70^{\circ} \mathrm{C}$.

Each sample in the HIV-1 test panels was analyzed in quadruplicate with the ultrasensitive and the standard method, to compare assay sensitivity.

All subjects were studied under research protocols approved by institutional review board of the Sahlgrenska Academy. Informed verbal consent was obtained from all patients.

Ultra-centrifugation: For the HIV-1 RNA panel, diluted plasma samples $(10 \mathrm{ml})$ were concentrated by ultra-centrifugation at $50,000 \times \mathrm{g}$ at $4{ }^{\circ} \mathrm{C}$ for 60 minutes. The pellet was resuspended in $1 \mathrm{ml}$ of HIV-1 negative plasma resulting in a 10 -fold concentration of the original sample. The suspension was vortexed and transferred to PCR tubes, and stored in $-70{ }^{\circ} \mathrm{C}$. No internal standard for the ultra-centrifugation step was used. In the clinical samples, 6 to $9 \mathrm{ml}$ of plasma was used, dependent on the amount of plasma available.

RT-PCR assay: Except for the ultra-centrifugation step, all samples were handled in the same way, and analyzed with AS-CT2 according to the manufacturer's instructions. Limit of quantification for AS-CT2 is reported to be 20 copies/ml and an internal control for the sample preparation procedure and successful PCR am- plification was added to every sample (HIV-1 Quantitation Standard).

Realtime PCR for quantification of total HIV-1 DNA: We used a single-step real time PCR to quantify total HIV-1 DNA in $25 \mu \mathrm{l}$ of PCR reaction mix containing $13 \mu \mathrm{l}$ of TaqMan Universal PCR Master Mix, $5 \mu$ l of chromosomal HIV-1 DNA and primers and probes as described below. We used a previously described protocol, except that we used half the sample size per reaction mix and another set of primers and probes [34,35]. Cycling conditions: $10 \mathrm{~min}$ activation step at $95{ }^{\circ} \mathrm{C}$ followed by $15 \mathrm{~s}$ at $95{ }^{\circ} \mathrm{C}$ and $60 \mathrm{~s}$ at $62{ }^{\circ} \mathrm{C}$ for 45 cycles. We used a primer-probe set for HIV-1 DNA quantification designed to bind to the conserved region of LTR: primers 5'-TTAAGCCTCAATAAAGCTTGCC-3', and 5'-GTTCGGGCGCCACTGCTAGA-3' with probe 5'FAM-CCAGAGTCACACAACAGAGGGGCA-3'TAMRA.

The clinical samples for the correlation study were analyzed at AIDS Research Institute, IrsiCaixa Foundation, Hospital Universario Germans Trias i Pujol, Barcelona, Spain. Sensitivity test and intra/inter-assay runs were performed at the Department of Clinical Virology, Gothenburg, Sweden.

Generation of a total HIV-1 DNA standard: We used a standard curve prepared by amplification of quantities ranging from 10 to $10^{6}$ copies of plasmid pCR2.1 harbouring LTR-LTR junction and the CCR5 human gene. The plasmid was kindly provided by Dr Mario Stevenson. The following primers and probes were used: primers CCR5 5'-GCTGTGTTGCGTCTCTCCCAGGA-3', 5'-CTCACAGCCCTGTGCCTCTTCTTC-3', primers LTR 5'-TTAAGCCTCAATAAAGCTTGCC-3', 5'-GTTCGGGCGCCACTGCTAGA-3'

and probes CCR5 5'FAM-AGCAGCGGCAGGACCAGCCCCAAG-TAMRA-3', LTR 5'FAM-CCAGAGTCACACAACAGAG-

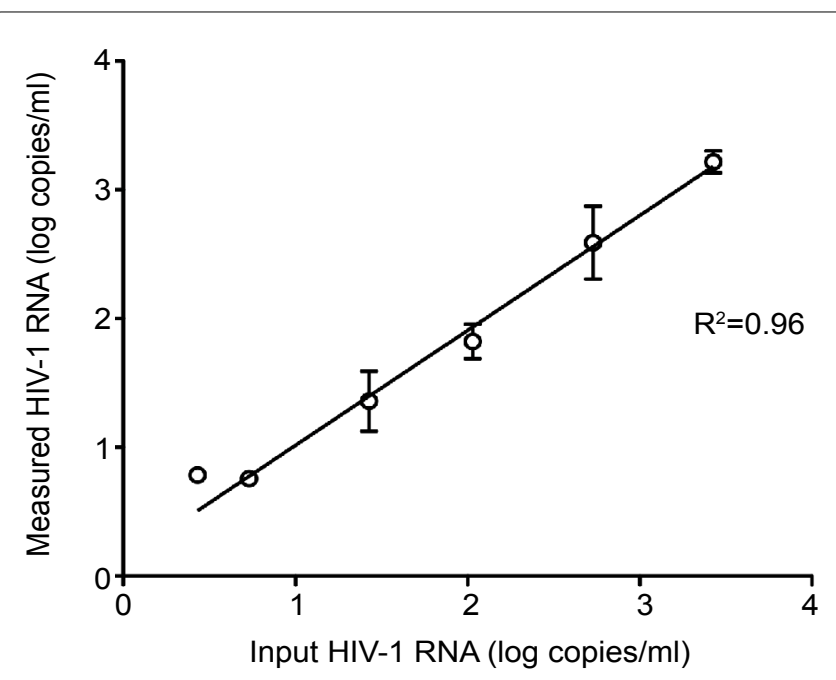

Figure 1: Linearity for measurements with ultrasensitive HIV-1 RNA PCR assay. In-put levels represent theoretical concentrations calculated from dilution of a plasma sample with a HIV-1 RNA level determined by COBAS Ampliprep/ COBAS Taqman HIV-1 test, version 2.0. All samples were run in quadruplicate, once in each dilution step. 
GGGCA-3'TAMRA. Reproducibility and limit of detection. To evaluate the reproducibility of the real time PCR assay for quantification of total HIV-1 DNA, we performed intra-assay and inter-assay tests using a clinical sample of known high plasma viral load. We used geometric Coefficient of Variation (CV) to estimate dispersion [36].

The limit of detection was determined by using a clinical sample in a serial dilution with lymphocytes from a healthy donor.

\section{Results}

Linearity of HIV-1 RNA quantification by the ultrasensitive assay: The assay accurately quantified a range of HIV-1 RNA concentrations obtained by serial dilution (Figure 1).

HIV-1 RNA sensitivity: The ultrasensitive assay showed greater sensitivity and was able to detect and quantify HIV-

Table 2: Comparison of The COBAS Ampliprep/COBAS Taqman HIV-1 Test v2 (Standard assay) with an ultra-centrifugation step prior to the standard protocol (Ultrasensitive assay) in two separate dilution series (Panel 1 and 2). Input copies/ml are theoretical concentrations calculated from a dilution series originated from a plasma sample quantified by COBAS Ampliprep/ COBAS Taqman HIV-1 Test, version 2. All samples were run in quadruplicate (1-4), once in each dilution step.

\begin{tabular}{|c|c|c|c|c|c|c|c|c|c|c|c|c|c|c|c|c|}
\hline \multirow{2}{*}{\begin{tabular}{|l} 
Panel 1 \\
Input \\
copies/ml
\end{tabular}} & \multicolumn{8}{|c|}{ Standard assay } & \multicolumn{8}{|c|}{ Ultrasensitive assay } \\
\hline & \multicolumn{2}{|c|}{1} & 2 & 3 & 4 & $\begin{array}{l}\text { Mean } \\
\text { copies/ml }\end{array}$ & SD & $\mathrm{CV}$ & 1 & 2 & 3 & \multicolumn{2}{|l|}{4} & $\begin{array}{l}\text { Mean } \\
\text { copies/ml }\end{array}$ & SD & $\mathrm{CV}$ \\
\hline 2683 & \multicolumn{2}{|c|}{3284} & 2332 & 3599 & 3915 & 3283 & 684 & $21 \%$ & 1942 & 1862 & 125 & \multicolumn{2}{|c|}{1639} & 1675 & 307 & $18 \%$ \\
\hline 537 & \multicolumn{2}{|c|}{686} & 660 & 572 & 637 & 639 & 49 & $8 \%$ & 554 & 607 & 459 & \multicolumn{2}{|c|}{148} & 442 & 205 & $46 \%$ \\
\hline 107 & \multicolumn{2}{|c|}{130} & 126 & 132 & 84 & 118 & 23 & $19 \%$ & 94 & 52 & 61 & \multicolumn{2}{|c|}{-} & 69 & 22 & $32 \%$ \\
\hline 26.8 & \multicolumn{2}{|c|}{$<20$} & $<20$ & 23 & $<20$ & - & - & & 24 & 47 & 20 & \multicolumn{2}{|c|}{13} & 26 & 15 & $57 \%$ \\
\hline 5.4 & \multicolumn{2}{|c|}{$<20$} & - & 30 & $<20$ & - & - & & 5.4 & 6.2 & 5.6 & \multicolumn{2}{|l|}{-} & 5.7 & 0.4 & $7 \%$ \\
\hline 2.7 & \multicolumn{2}{|c|}{$<20$} & - & - & $<20$ & - & - & & Det & Det & 6.1 & \multicolumn{2}{|c|}{ Det } & - & - & \\
\hline \multicolumn{9}{|l|}{ Panel 2} & \multicolumn{8}{|c|}{ Ultrasensitive assay } \\
\hline \multicolumn{2}{|c|}{ Input copies/ml } & 1 & 2 & 3 & 4 & Mean copies/ml & SD & $\mathrm{CV}$ & 1 & 2 & 3 & 4 & \multicolumn{2}{|c|}{ Mean copies/ml } & SD & CV \\
\hline 27 & & 50 & 36 & 200 & 78 & 91 & 75 & $82 \%$ & 103 & 105 & 104 & 59 & \multicolumn{2}{|c|}{93} & 23 & $24 \%$ \\
\hline 9 & & $<20$ & 44 & $<20$ & 29 & 36.5 & 11 & $29 \%$ & 32 & 27 & 43 & 24 & \multicolumn{2}{|l|}{31} & 8.3 & $27 \%$ \\
\hline 3 & & - & - & - & $<20$ & & & & - & 14 & 5.1 & 7 & \multicolumn{2}{|l|}{8.7} & 4.7 & $54 \%$ \\
\hline 1 & & - & - & - & - & & & & 3.2 & - & 2.1 & 2.2 & \multicolumn{2}{|l|}{2.5} & 0.6 & $24 \%$ \\
\hline
\end{tabular}

SD: Standard Deviation; CV: Coefficient of Variation; Det: Detected but not quantified by the ultrasensitive assay; <20: Detected but not quantified by the standard assay (The COBAS Taqman HIV-1 test, version 2.0 from Roche).

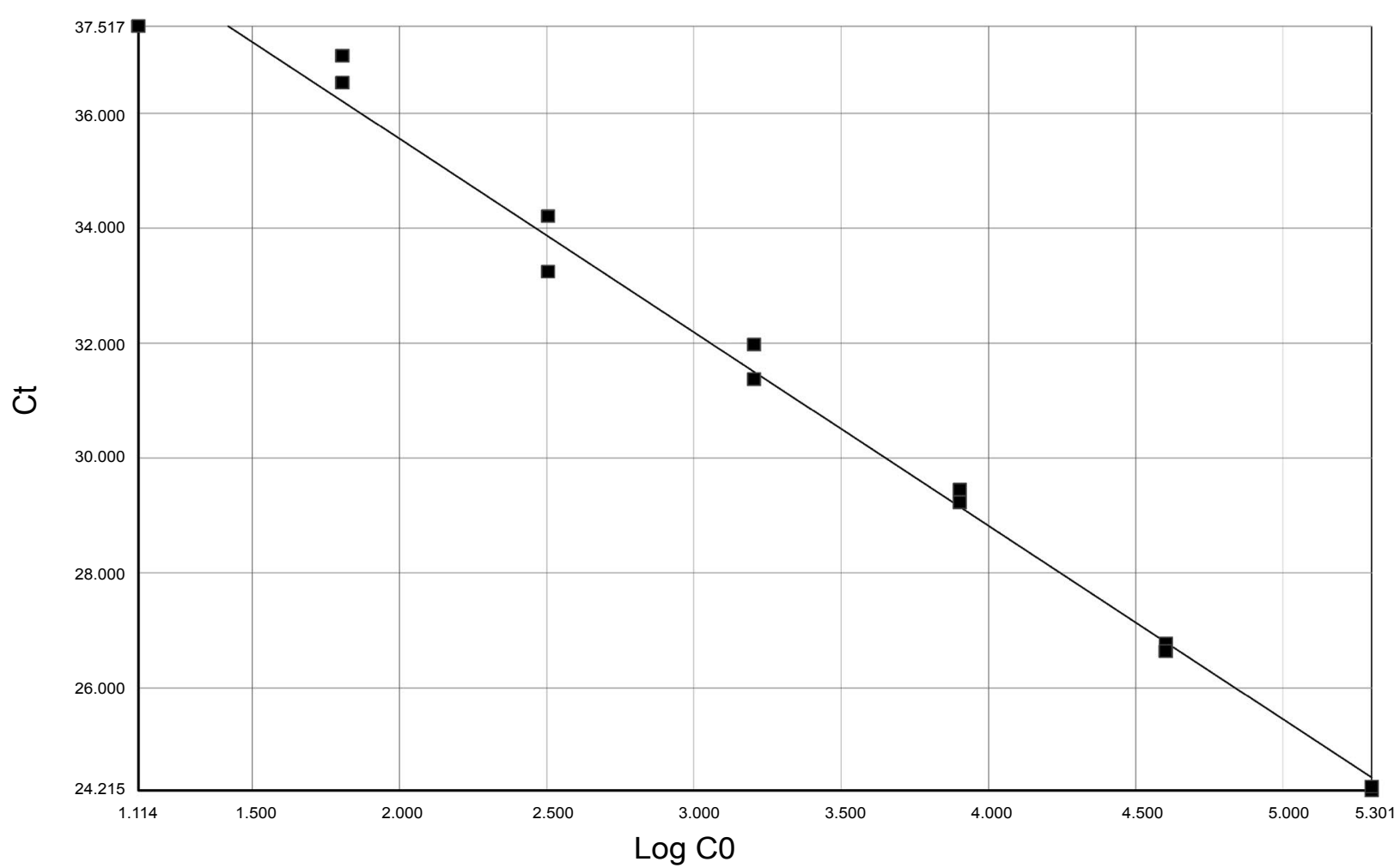

Detector: LTRPU, Slope:-3.368374, Intercept: 42.294720, R2: 0.989536

Figure 2: Standard curve generated by plasmid pCR2.1 showing slope $(-3.37)$ and linear regression $\left(R^{2}=0.99\right)$ for $L T R$. Ct = Cycle threshold. Log CO = Log LTR. 
1 RNA at lower plasma concentrations than the standard assay (Table 2). In the first panel the ultrasensitive method failed to quantify HIV-1 RNA at 2.7 copies $/ \mathrm{ml}$ in three out of four samples, but the assay detected HIV-1 RNA in all the wells at this dilution step (Table 2, panel 1). To further evaluate assay sensitivity, a second dilution series with a lower range of estimated copy numbers was created. In this panel, the ultrasensitive assay consistently detected HIV-1 RNA in dilutions estimated to contain 3 copies $/ \mathrm{ml}$ and $1 \mathrm{copy} / \mathrm{ml}$ (in three out of four wells at both dilution steps). The mean value for the obtained concentration was 2.5 copies $/ \mathrm{ml}$ (SD 0.6 copies $/ \mathrm{ml}$ ) at the 1 copy $/ \mathrm{ml}$ input level (Table 2, panel 2).

Reproducibility and limit of detection for quantification of total HIV-1 DNA: Figure 2 and Figure 3 show slopes (-3.37 for LTR and -3.34 for CCR5) and linear regression for both LTR and CCR5 genes $\left(R^{2}=0.99\right.$ respectively) for plasmid pCR2.1.

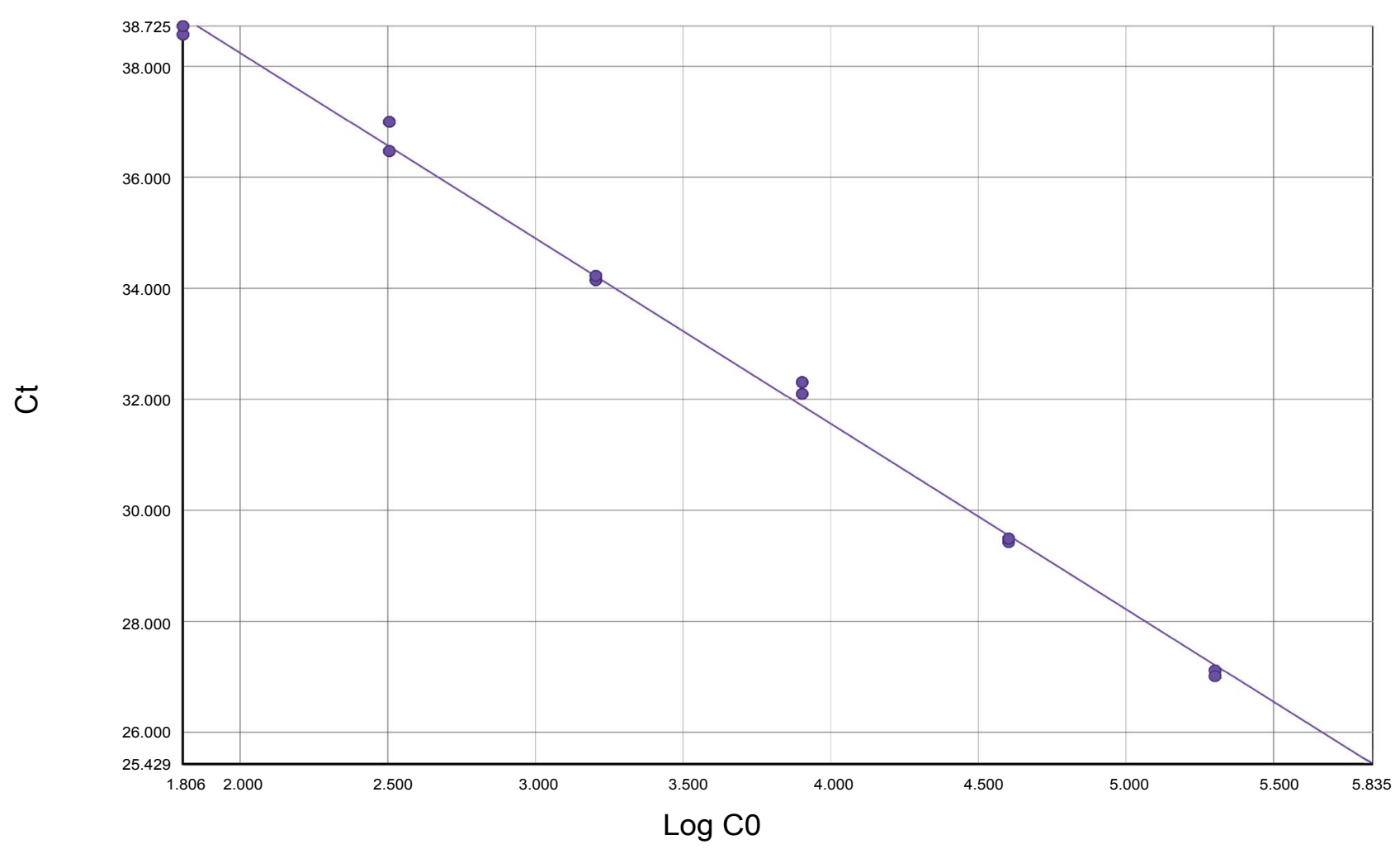

Detector: CCR5, Slope: -3.340354, Intercept: 44.921692, R2: 0.996709

Figure 3: Standard curve generated by plasmid pCR2.1 showing slope $(-3.34)$ and linear regression $\left(R^{2}=0.99\right)$ for human genome CCR5. $\mathrm{Ct}=$ Cycle threshold. Log C0 = Log CCR5.

Table 3: Dilution series for determining limit of quantification of total HIV-1 DNA PCR assay.

\begin{tabular}{|c|c|c|c|c|c|c|c|}
\hline Dilution & HIV-1 DNA/million cells & HIV-1 DNA/million cells mean & HIV-1 DNA/million cells SD & CV & $\mathbf{C t}$ & Ct mean & Ct SD \\
\hline $1: 1$ & 44 & 37 & 9.9 & $26 \%$ & 38.7 & 39.0 & 0.3 \\
\hline $1: 1$ & 26 & & & & 39.4 & & \\
\hline $1: 1$ & 42 & & & & 39.0 & & \\
\hline $1: 2$ & 20 & 14 & 7.1 & $49 \%$ & 40.4 & 41.0 & 0.9 \\
\hline $1: 2$ & 9.4 & & & & 41.6 & & \\
\hline $1: 2$ & - & & & & - & & \\
\hline $1: 4$ & 17 & 10 & 8.8 & $84 \%$ & 40.6 & 41.6 & 1.5 \\
\hline $1: 4$ & 4.2 & & & & 42.7 & & \\
\hline $1: 4$ & - & & & & - & & \\
\hline 1:6 & 12 & 7.8 & 3.8 & $50 \%$ & 43 & 43.7 & 0.6 \\
\hline $1: 6$ & 6.0 & & & & 44.0 & & \\
\hline $1: 6$ & 5.1 & & & & 44 & & \\
\hline $1: 8$ & 3.4 & 3.1 & 1.6 & $50 \%$ & 43.8 & 43.9 & 1.0 \\
\hline $1: 8$ & 1.4 & & & & 44.9 & & \\
\hline 1:8 & 4.6 & & & & 43.0 & & \\
\hline
\end{tabular}

SD: Standard Deviation; CV: Coefficient of Variation. 
The geometric $\mathrm{CV}$ in five replicates for the intra-assay test was $13.2 \%$ and for the inter-assay test the geometric $\mathrm{CV}$ in five runs of duplicates was $23.2 \%$ (Figure 4). The assay detected and quantified HIV-1 DNA down to the range of 1.4 to 4.6 copies/million cells suggesting the limit of quantification to 3 copies/million cells (Table 3 ).

Clinical assay performances and correlation of total HIV1 DNA and plasma HIV-1 RNA: In samples from 19 HIV-1 positive patients measured by AS-CT2, 14 had non-detect- able plasma HIV-1 RNA levels, three had detectable but not quantifiable levels and two patients had 24 copies $/ \mathrm{ml}$ and 45 copies $/ \mathrm{ml}$ respectively. HIV-1 RNA was detected in 14 out of 19 samples by the ultrasensitive method. In 9 of these 14 positive samples, HIV-1 RNA was quantified in the range of 2.3 to 34.5 copies/ml. The HIV-1 RNA strains detected had previously been identified as subtype B in 9 cases, subtype $C$ in 1 , subtype CRF-AE in 2, subtype $D$ in 1 , and 1 had been determined as probably subtype $F($ Table 1 ).
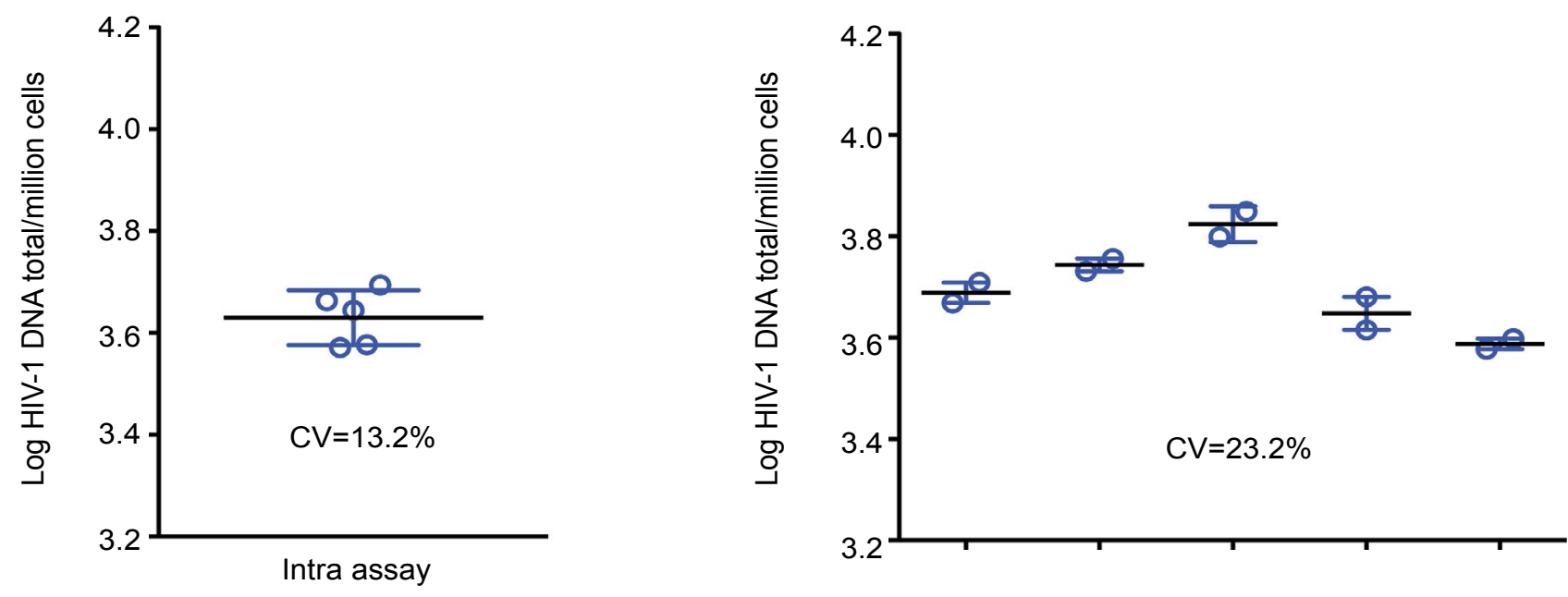

Intra assay

Figure 4: Total HIV-1 DNA PCR intra- and inter-assay validation. Intra-assay: mean log HIV-1 DNA was 3.63/million cells (4291 copies/million cells) in five replicates, SD log HIV-1 DNA 0.054/million cells, geometric CV 13.2\%. Inter-assay: mean log HIV-1 DNA in five runs of duplicates was 3.70/million cells (5088 copies/million cells), SD log HIV-1 DNA 0.09/million cells, geometric CV $23.2 \%$.

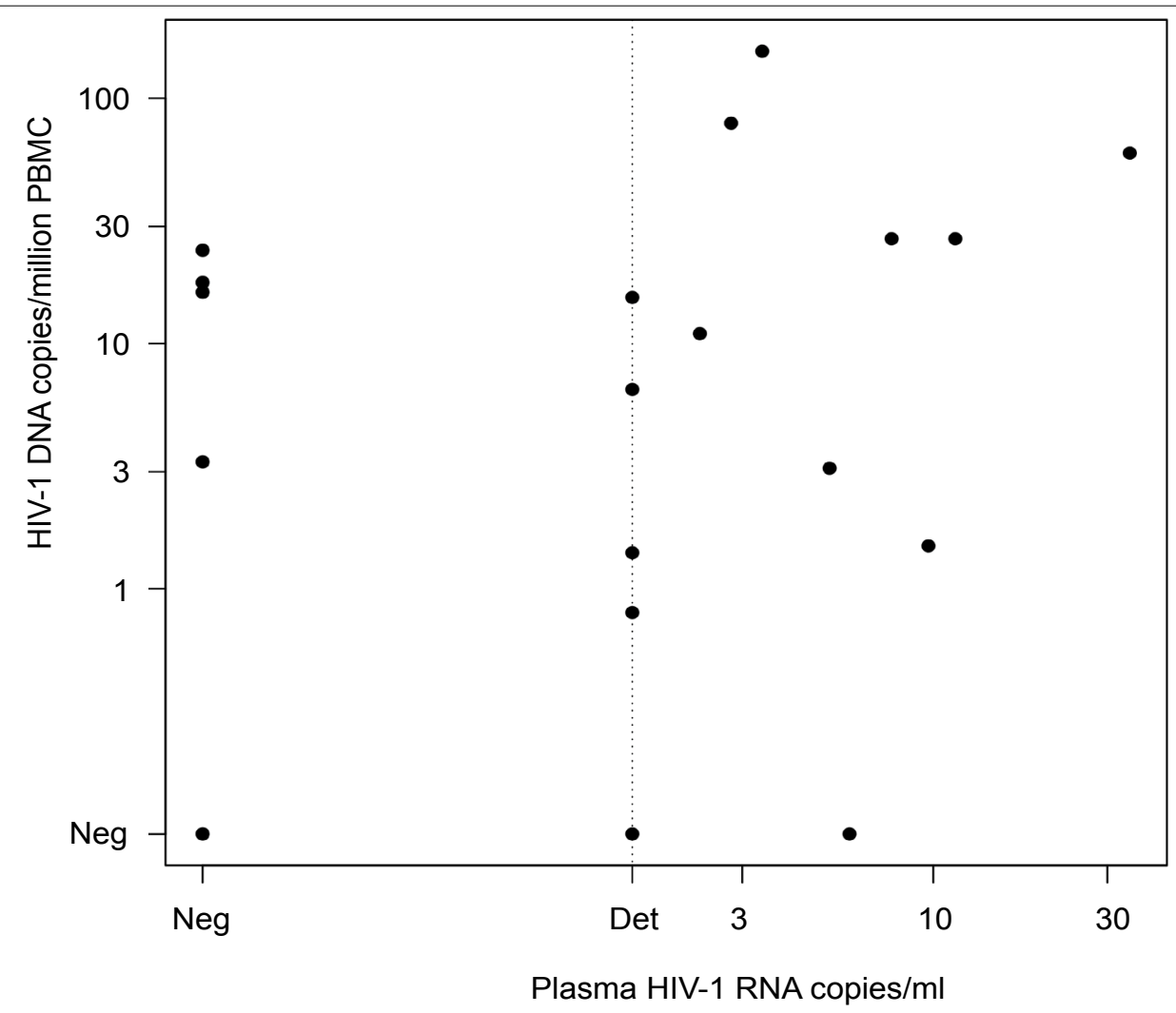

Figure 5: Correlation of total HIV-1 DNA copies/million cells in PBMC and HIV-1 plasma RNA copies/ml in 19 patients with longterm suppressive cART. No significant correlation was seen $(p=0.28$, Spearman $r=0.26)$. Det $=$ detected but not quantified. Neg $=$ negative. 
HIV-1 DNA was detected in 16 out of 19 samples and was quantified at levels ranging from 0.8 to 155.6 copies/million cells. The assay detected ten subtype B strains, four subtype $C$, one subtype $D$ and one probable subtype F strain (Table 1). HIV-1 DNA was not detected in any of the subtype CRF-AE strains. No correlation was found between plasma HIV-1 RNA and total viral HIV-1 DNA $(p=0.29, r=0.28)$ (Figure 5).

\section{Discussion}

In this study, we present a sensitive and subtype independent quantification of plasma HIV-1 RNA, and a real-time PCR for quantification of total HIV-1 DNA. By adding an ultra-centrifugation step prior to the COBAS Taqman HIV-1 test, version 2.0, we obtained increased sensitivity, quantifying HIV-1 RNA down to 3 copies $/ \mathrm{ml}$ plasma. In our study we did not attempt to concentrate virus from more than a maximum $10 \mathrm{ml}$ of plasma, but a larger volume might have lowered the limit of quantification in the assay further. There is a considerably large variation when comparing the two sensitivity panels, around $0.5 \mathrm{log}$. This variation could be expected but would probably be reduced by a larger number of panels.

The previously described single copy assay has the disadvantage of being labour intensive and is developed only for detection of subtype B strains [14]. Other reports have described successful modifications of the Abbot Real Time assay and the Amplicor Ultrasensitive assays, including an ultra-centrifugation step and increased sample volumes [19,21], but did not evaluate amplification of different subtypes. To our knowledge this is the first report of an ultrasensitive detection of various subtypes by a modified commercial HIV-1 RNA assay. The assay showed good clinical performance, detecting residual viremia in all subtypes tested. HIV-1 RNA was not detected by the ultrasensitive assay in five samples, and may indeed reflect true negative samples. It should be pointed out that we used smaller plasma volumes in the clinical performance test (due to limited access) than in the sensitivity panel ( 6 to $9 \mathrm{ml}$ compared to $10 \mathrm{ml}$ ), which has an impact on the limit of detection. Although the number of samples was small, our results indicate that the ultrasensitive assay could be used for measurements of residual viremia in various HIV-1 subtypes.

We also evaluated quantification of total HIV-1 DNA in PBMC by real time PCR and found good reproducibility and sensitivity, with a suggested limit of quantification to 3 copies/million cells and geometric intra-and inter-assay CVs of $13.2 \%$ and $23.2 \%$ respectively. The assay quantified total HIV-1 DNA in all subtypes tested except CRF_AE. This was probably due to primer mismatch since none of the CRF_AE samples were detected by the HIV-1 DNA assay. One subtype B strain was not detected by the HIV-1 DNA assay, which was probably due to very low viral levels since this sample was also not detected by the HIV-1 RNA assay.
When comparing total HIV-1 DNA in PBMC and plasma HIV-1 RNA in patients on suppressive treatment, no significant correlation was found. A correlation between total HIV-1 DNA in PBMC and plasma residual viremia (HIV-1 RNA) would indicate that a major part of the viremia is derived from resting $\mathrm{CD}^{+} \mathrm{T}$-cells, and might indicate that a combination of these assays could be used to estimate the reservoir size. One previous study reports a correlation between plasma residual viremia and the frequency of $\mathrm{CD}^{+}{ }^{+}$T-cells carrying HIV-1 DNA (measured by total HIV-1 DNA). In this study however, nearly $40 \%$ of the study participants with undetectable plasma viremia exhibited a relatively high frequency of HIV-1 infection in the $\mathrm{CD}^{+} \mathrm{T}$-cell compartment. Furthermore, no correlation between activation markers and plasma viremia was found, indicating the possibility of measurement of non-productive virions [37]. However, in a more recent study, Gandhi, et al. showed that residual viremia was associated with IUPM in patients on suppressive cART [38]. Buzon, et al. showed a correlation between the frequency of cells with replication-competent HIV-1, and the total amount of HIV-1 DNA in suppressed patients with treatment initiated at different phase of the infection. However, in this study, residual viremia did not seem to be dependent on the levels of HIV-1 DNA [39].

The lack of correlation in this study could be due to lack of precision and sensitivity of the assays used in the very low levels of HIV-1 RNA and HIV-1 DNA that were present in the tested samples. Residual viremia is usually estimated to around 3 HIV-1 RNA copies $/ \mathrm{ml}$ and below $[13,40]$ and stochastic influences might have influenced the obtained concentrations. We also had a small sample size, although a larger sample size might not have changed the outcome of this particular result. Alternative explanations may be productive viral reservoirs other than latently infected $\mathrm{CD} 4^{+}$T-cells [41-43]. Low levels of replication and cell-to-cell spread of virus in other compartments may not be reflected in the levels of plasma residual viremia, even as measured by the most sensitive assays.

Recent studies have also questioned the use of PCR assays to determine reservoir size as they do not differentiate between replication-competent proviral HIV-1 DNA and incomplete HIV-1 DNA that cannot produce virions [44]. However, PCR measurements are less costly and labour-intensive to perform than for example IUPM, and there are studies proposing these techniques as surrogate measures of reservoir size [31-33]. Previous studies have suggested that measurement of both total and integrated HIV-1 DNA may be important in estimating reservoir size [32]. In our material, the patients were treated for at least one year prior to sampling and most of the patients were successfully treated for several years. This timeframe should be sufficient for the eradication of other forms of HIV-1 DNA than the integrated pro-viral genome [31]. Again, many of the integrated pro-viruses may not be vi- 
able. There are also reports indicating that some patients may have an excess of unintegrated forms of HIV-1 DNA despite long-term cART [45].

This study has some limitations. We did not incorporate an internal standard in the ultrasensitive HIV-1 PCR to monitor the recovery rate of HIV-1 RNA through the ultra-centrifugation and extraction procedures. Furthermore, control copies in the reactions were estimated through PCR, which introduces some uncertainty in the control dilution step. As mentioned above, we also had a limited number of clinical samples tested and future studies, incorporating more samples with diverse subtypes will evaluate this assay further.

In conclusion: by modifications of a commercial assay, ultrasensitive quantification of plasma HIV-1 RNA down to 3 copies $/ \mathrm{ml}$ was achieved, and quantification of total HIV-1 DNA in PBMC in the range of 3 copies/ million cells by real time PCR was documented. The HIV1 DNA assay need to be modified to incorporate also CRF subtypes and need further testing considering the small sample size. However, the assays were subtype independent to a large extent, making them suitable for research and possibly also clinical use in settings where a variety of HIV-1 subtypes are present.

\section{Acknowledgments}

This work was supported by the Swedish Research Council (K2008-58P-20930-01-4), the Sahlgrenska Academy at the University of Gothenburg (ALFGBG-141741), and Gothenburg Medical Society.

\section{Declaration of Interest}

Tomas Mellberg, Jon Krabbe, Maria J. Buzon, Ulrika Noborg, Staffan Lindh, Staffan Nilsson and Bo Svennerholm have all declared that no competing interests exist. Magnus Gisslén has the following potential conflicts: Received honorarium from Abbvie, BMS, Gilead, GSK/ ViiV, and Janssen for participation on scientific advisory boards and educational lectures.

\section{References}

1. Gulick RM, Mellors JW, Havlir D, Eron JJ, Gonzalez C, et al. (1997) Treatment with indinavir, zidovudine, and lamivudine in adults with human immunodeficiency virus infection and prior antiretroviral therapy. N Engl J Med 337: 734-739.

2. Perelson AS, Essunger $P, C a o Y$, Vesanen $M$, Hurley $A$, et al. (1997) Decay characteristics of HIV-1-infected compartments during combination therapy. Nature 387: 188-191.

3. Chun TW, Engel D, Berrey MM, Shea T, Corey L, et al (1998) Early establishment of a pool of latently infected, resting CD4 (+) T cells during primary HIV-1 infection. Proc Natl Acad Sci U S A 95: 8869-8873.

4. Chun TW, Stuyver L, Mizell SB, Ehler LA, Mican JA, et al. (1997) Presence of an inducible HIV-1 latent reservoir during highly active antiretroviral therapy. Proc Natl Acad Sci U S A 94: 13193-13197.

5. Finzi D, Hermankova M, Pierson T, Carruth LM, Buck C, et al. (1997) Identification of a reservoir for HIV-1 in patients on highly active antiretroviral therapy. Science 278: 12951300.

6. Doyle T, Smith C, Vitiello P, Cambiano V, Johnson M, et al. (2012) Plasma HIV-1 RNA detection below 50 copies/ $\mathrm{ml}$ and risk of virologic rebound in patients receiving highly active antiretroviral therapy. Clin Infect Dis 54: 724-732.

7. Maggiolo F, Callegaro A, Cologni G, Bernardini C, Velenti D, et al. (2012) Ultrasensitive assessment of residual low-level HIV viremia in HAART-treated patients and risk of virological failure. J Acquir Immune Defic Syndr 60: 473482.

8. Ruelle J, Debaisieux L, Vancutsem E, De Bel A, Delforge ML, et al. (2012) HIV-1 low-level viraemia assessed with 3 commercial real-time PCR assays show high variability. BMC Infectious Diseases 12: 100.

9. Dahl V, Peterson J, Spudich S, Lee E, Shacklett BL, et al. (2013) Single-copy assay quantification of HIV-1 RNA in paired cerebrospinal fluid and plasma samples from elite controllers. AIDS 27: 1145-1149.

10. Dahl V, Peterson J, Fuchs D, Gisslen M, Palmer S, et al. (2014) Low levels of HIV-1 RNA detected in the cerebrospinal fluid after up to 10 years of suppressive therapy are associated with local immune activation. AIDS 28: 2251-2258.

11. Markowitz M, Evering TH, Garmon D, Caskey M, La Mar M, et al. (2014) A randomized open-label study of 3- versus 5-drug combination antiretroviral therapy in newly HIV-1-infected individuals. J Acquir Immune Defic Syndr 66: 140-147.

12. Gandhi RT, Zheng L, Bosch RJ, Chan ES, Margolis DM, et al. (2010) The effect of raltegravir intensification on low-level residual viremia in HIV-infected patients on antiretroviral therapy: a randomized controlled trial. PLoS Medicine 8.

13. Palmer S, Maldarelli F, Wiegand A, Bernstein B, Hanna GJ, et al. (2008) Low-level viremia persists for at least 7 years in patients on suppressive antiretroviral therapy. Proc Natl Acad Sci U S A 105: 3879-3884.

14. Palmer S, Wiegand AP, Maldarelli F, Bazmi H, Mican JM, et al. (2003) New real-time reverse transcriptase-initiated PCR assay with single-copy sensitivity for human immunodeficiency virus type 1 RNA in plasma. J Clin Microbiol 41: 4531-4536.

15. Graf T, Pinto AR (2013) The increasing prevalence of HIV-1 subtype $C$ in Southern Brazil and its dispersion through the continent. Virology 435: 170-178.

16. Neogi $U$, Haggblom A, Santacatterina M, Bratt G, Gisslen M, et al. (2014) Temporal trends in the Swedish HIV-1 epidemic: increase in non-B subtypes and recombinant forms over three decades. PLoS One 9: e99390.

17. UK Collaborative Group on HIV Drug Resistance (2014) The increasing genetic diversity of HIV-1 in the UK, 20022010. AIDS 28: 773-780.

18. Siemieniuk RA, Beckthold B, Gill MJ (2013) Increasing HIV subtype diversity and its clinical implications in a sentinel North American population. Can J Infect Dis Med Microbiol 24: 69-73.

19. Havlir DV, Bassett R, Levitan D, Gilbert P, Tebas $P$, et al. (2001) Prevalence and predictive value of intermittent viremia with combination hiv therapy. JAMA 286: 171-179.

20. Dornadula G, Zhang H, VanUitert B, Stern J, Livornese L, et al. (1999) Residual HIV-1 RNA in blood plasma of patients taking suppressive highly active antiretroviral therapy. JAMA 282: 1627-1632.

21. Yukl SA, Li P, Fujimoto K, Lampiris H, Lu CM, et al. (2011) Modification of the Abbott RealTime assay for detection of 
HIV-1 plasma RNA viral loads less than one copy per milliliter. J Virol Methods 175: 261-265.

22. Paba P, Fabeni L, Ciccozzi M, Perno CF, Ciotti M (2011) Performance evaluation of the COBAS/TaqMan HIV-1 v2.0 in HIV-1 positive patients with low viral load: a comparative study. J Virol Methods 173: 399-402.

23. Erali M, Hillyard DR (1999) Evaluation of the ultrasensitive Roche Amplicor HIV-1 monitor assay for quantitation of human immunodeficiency virus type 1 RNA. J Clin Microbiol 37: $792-795$

24. Archin NM, Cheema M, Parker D, Wiegand A, Bosch RJ, et al. (2010) Antiretroviral intensification and valproic acid lack sustained effect on residual HIV-1 viremia or resting CD4+ cell infection. PLoS One 5: e9390.

25. Archin NM, Liberty AL, Kashuba AD, Choudhary SK, Kuruc JD, et al. (2012) Administration of vorinostat disrupts HIV1 latency in patients on antiretroviral therapy. Nature 487: 482-485

26. Lindkvist A, Eden A, Norstrom MM, Gonzalez VD, Nilsson $S$, et al. (2009) Reduction of the HIV-1 reservoir in resting CD4+ T-lymphocytes by high dosage intravenous immunoglobulin treatment: a proof-of-concept study. AIDS Res Ther 6: 15

27. Mellberg T, Gonzalez VD, Lindkvist A, Eden A, Sonnerborg A, et al. (2011) Rebound of residual plasma viremia after initial decrease following addition of intravenous immunoglobulin to effective antiretroviral treatment of HIV. AIDS Res Ther 8: 21.

28. Xing S, Bullen CK, Shroff NS, Shan L, Yang HC, et al. (2011) Disulfiram reactivates latent HIV-1 in a Bcl-2-transduced primary CD4+ T cell model without inducing global $T$ cell activation. J Virol 85: 6060-6064.

29. Siliciano JD, Siliciano RF (2005) Enhanced culture assay for detection and quantitation of latently infected, resting CD4+ T-cells carrying replication-competent virus in HIV-1-infected individuals. Methods Mol Biol 304: 3-15.

30. Ho YC, Shan L, Hosmane NN, Wang J, Laskey SB, et al. (2013) Replication-competent noninduced proviruses in the latent reservoir increase barrier to HIV-1 cure. Cell 155: 540-551.

31. Koelsch KK, Liu L, Haubrich R, May S, Havlir D, et al. (2008) Dynamics of total, linear nonintegrated, and integrated HIV1 DNA in vivo and in vitro. $J$ Infect Dis 197: 411-419.

32. Mexas AM, Graf EH, Pace MJ, Yu JJ, Papasavvas E, et al. (2012) Concurrent measures of total and integrated HIV DNA monitor reservoirs and ongoing replication in eradication trials. AIDS 26: 2295-2306.

33. Vandergeeten C, Fromentin R, Merlini E, Lawani MB, DaFonseca S, et al. (2014) Cross-clade ultrasensitive PCRbased assays to measure HIV persistence in large-cohort studies. J Virol 88: 12385-12396.
34. Brussel A, Sonigo P (2003) Analysis of early human immunodeficiency virus type 1 DNA synthesis by use of a new sensitive assay for quantifying integrated provirus. J Virol 77: 10119-10124.

35. Buzon MJ, Massanella M, Llibre JM, Esteve A, Dahl V, et al. (2010) HIV-1 replication and immune dynamics are affected by raltegravir intensification of HAART-suppressed subjects. Nat Med 16: 460-465.

36. Kirkwood T (1979) Geometric means and measures of dispersion. Biometrics, 908-909.

37. Chun TW, Murray D, Justement JS, Hallahan CW, Moir S, et al. (2011) Relationship Between Residual Plasma Viremia and the Size of HIV Proviral DNA Reservoirs in Infected Individuals Receiving Effective Antiretroviral Therapy. J Infect Dis 204: 135-138.

38. Gandhi RT, Bosch RJ, Aga E, Bedison MA, Bastow B, et al. (2013) Residual plasma viraemia and infectious HIV-1 recovery from resting memory CD4 cells in patients on antiretroviral therapy: results from ACTG A5173. Antivir Ther 18: $607-613$

39. Buzon MJ, Martin-Gayo E, Pereyra F, Ouyang Z, Sun H, et al. (2014) Long-term antiretroviral treatment initiated at primary HIV-1 infection affects the size, composition, and decay kinetics of the reservoir of HIV-1-infected CD4 T cells. J Virol 88: 10056-10065.

40. Maldarelli F, Palmer S, King MS, Wiegand A, Polis MA, et al. (2007) ART suppresses plasma HIV-1 RNA to a stable set point predicted by pretherapy viremia. PLoS Pathog 3 : e46.

41. Chun TW, Nickle DC, Justement JS, Meyers JH, Roby G, et al. (2008) Persistence of HIV in gut-associated lymphoid tissue despite long-term antiretroviral therapy. J Infect Dis 197: 714-720.

42. Yukl SA, Shergill AK, McQuaid K, Gianella S, Lampiris $H$, et al. (2010) Effect of raltegravir-containing intensification on HIV burden and T-cell activation in multiple gut sites of HIV-positive adults on suppressive antiretroviral therapy. AIDS 24: 2450-2459.

43. Bailey JR, Sedaghat AR, Kieffer T, Brennan T, Lee PK, et al. (2006) Residual human immunodeficiency virus type 1 viremia in some patients on Antiretroviral therapy is dominated by a small number of invariant clones rarely found in circulating CD4(+) T cells. J Virol 80: 6441-6457.

44. Eriksson S, Graf EH, Dahl V, Strain MC, Yukl SA, et al. (2013) Comparative analysis of measures of viral reservoirs in HIV-1 eradication studies. PLoS Pathog 9: e1003174.

45. Agosto LM, Liszewski MK, Mexas A, Graf E, Pace M, et al. (2011) Patients on HAART often have an excess of unintegrated HIV DNA: implications for monitoring reservoirs. Virology 409: 46-53. 